\title{
Muscle biopsy in polymyositis and dermatomyositis: a clinicopathological study
}

\author{
HANS A. SCHWARZ, GERARD SLAVIN,* PETER WARD,* AND \\ BARBARA M. ANSELL
}

From the Clinical Research Centre, Division of Rheumatology, and the *Department of Histopathology, Northwick Park Hospital, Harrow, Middlesex

SUMMARY The initial muscle biopsies of 30 patients with active polymyositis or dermatomyositis have been assessed histologically and morphometrically. Only 1 patient $(3 \cdot 3 \%)$ had a normal biopsy, while 3 had a normal electromyograph and 8 had a normal serum CPK level. No singificant morphological differences were found between the various diagnostic subgroups. Sequential biopsies of 11 of these patients treated either with corticosteroids only or with intensive immunosuppression were studied. With response to treatment atrophy factors, variability coefficients, internal nuclei count, and the various histological changes improved in the whole group of patients. However, there was no consistent correlation between any of the morphological features or between these and clinical parameters in individual patients. The percentage of fibres with internal nuclei in the initial biopsy was lower $(\mathrm{P}<0.001)$ in patients responding to up to $60 \mathrm{mg}$ prednisolone per day than in those who eventually required cytotoxic drugs in addition.

Muscle biopsy plays an important part in the diagnosis of polymyositis (PM) and dermatomysitis (DM). Open biopsy is still widely used, but needle biopsy is much less traumatic and has been claimed to yield very good results for most diagnostic purposes. ${ }^{1}$ In 2 recent large series ${ }^{2,3} 17 \%$ and $12 \cdot 5 \%$ respectively of the cases meeting clinical, electromyographic, and biochemical criteria for PM or DM have been reported to show no histological abnormalities in their biopsies. However, histochemical assessments were used to only a limited extent.

The sensitivity of the muscle biopsy is enhanced considerably by including histochemical and histomorphometric assessments. These parameters have been studied in a series of 18 patients $^{4}$ by means of open biopsy, with the morphometric results being expressed on a semiquantitative scale. We have examined needle biopsies of muscle in 30 patients with active PM or DM using histochemical and morphometric techniques and correlated the findings with clinical features.

While the majority of patients appear to respond to corticosteroids, some do not improve until the

Accepted for publication 30 August 1979.

Correspondence to Dr Barbara M. Ansell, Clinical Research Centre, Division of Rheumatology, Northwick Park Hospital, Watford Road, Harrow, Middlesex HA1 3UJ. addition of cytotoxic drugs. It is not possible to predict the response to steroids in the individual patient. Intensive immunosuppression with antilymphocyte globulin, aza hioprine, and corticosteroids has been suggested as a possible treatment in steroid-resistant cases. ${ }^{5}$

The effects of the drugs used in these diseases on muscle morphology are not well known, since the data available mainly originate from patients who were refractory to treatment. ${ }^{3,6}$ In order to evaluate needle biopsy of muscle as an additional tool for monitoring disease activity and treatment in PM and DM we have also studied the sequential biopsies of 11 patients, 5 treated with corticosteroids alone and 6 with the intensive immunosuppressive regimen. ${ }^{5}$

\section{Material and methods}

\section{CLINICAL DETAILS}

Between May 1973 and December 197830 patients (18 females, 12 males) aged 7 to 81 years with a diagnosis of active PM or DM have been seen at Northwick Park Hospital. Eighteen of these were first referrals. Using the criteria proposed by Bohan and Peter ${ }^{7}$ we made a definite diagnosis in 25 and probable in 5 cases. Their distribution among the diagnostic categories is shown in Table 1. 
Table 1 Distribution of 30 patients among the diagnostic subgroups ${ }^{7}$

\begin{tabular}{lcll}
\hline & No. & Sex & $\begin{array}{l}\text { Mean age at } \\
\text { onset }(y r)(\text { range })\end{array}$ \\
\hline PM & 9 & $3 \mathrm{M} ; 6 \mathrm{~F}$ & $51 \cdot 0(16-73)$ \\
DM & 5 & $1 \mathrm{M} ; 4 \mathrm{~F}$ & $37.4(19-56)$ \\
DM + malignancy & 4 & $1 \mathrm{M} ; 3 \mathrm{~F}$ & $53.7(44-70)$ \\
DM in childhood & 4 & $3 \mathrm{M} ; 1 \mathrm{~F}$ & $4.0(3-10)$ \\
Overlap syndromes* & 8 & $4 \mathrm{M} ; 4 \mathrm{~F}$ & $46.9(29-81)$ \\
Total & 30 & $12 \mathrm{M} ; 18 \mathrm{~F}$ & $42.1(3-81)$ \\
\hline
\end{tabular}

*Associated disease: scleroderma 6; SLE 1; hypogammaglobulinaemia 1.

The patients with malignancies all had DM. The 3 females suffered from carcinoma of the breast; the male had a transitional cell carcinoma of the ureter. The malignancies were detected 3-16 months before onset of the muscle symptoms, but in 1 patient scleroderamtous skin lesions preceded the carcinoma of the breast by 2 years. In the other patient with DM the skin lesions appeared at the time of the muscle symptoms.

The 4 children showed the typical rash of DM; 3 had subcutaneous calcinosis.

The mean duration of muscle symptoms before the initial biopsy was 15.6 months (1-60), with a mean of 6.5 months (1-23) in the 18 primarily referred patients and 29.7 months $(2-60)$ in the 12 secondary referrals. Ten patients had been on corticosteroids (mean $19 \mathrm{mg}$ per day; range $2 \cdot 5-80 \mathrm{mg}$ ) for various lengths of time, usually a few months. The findings on admission are listed in Table 2.

One or more subsequent biopsies have been taken in a group of 11 patients whose clinical data are given in Table 3. As they formed part of a larger study in intensive immunosuppression in connective tissue diseases they were allocated at random to either corticosteroids only, starting usually with $60 \mathrm{mg}$ prednisolone per day, or to a regimen consisting of antilymphocyte globulin (750 mg daily for 15 days), azathioprine ( $3 \mathrm{mg} / \mathrm{kg}$ per day up to $150 \mathrm{mg}$ per day), and prednisolone $(150 \mathrm{mg}$ per day, reduced over 10 days to a maintenance level according to the clinical situation). ${ }^{5}$

Table 2 Admission state

\begin{tabular}{lr}
\hline Muscles $(\mathrm{n}=30)$ & 30 \\
Symmetrical proximal weakness & 9 \\
Pain and/or tenderness & 8 \\
Dysphagia & 27 \\
Biopsy $(\mathrm{n}=30)$ & 2 \\
'Myositic' & \\
Atrophy only & 25 \\
$E M G(\mathrm{n}=28)$ & 22 \\
'Myositic' & 2 \\
CPK $(\mathrm{n}=30)$ & \\
Raised & \\
Raised only later on & \\
\hline
\end{tabular}

Table 3 Patients in therapeutic study with sequential muscle biopsies after an interval of at least 4 months

\begin{tabular}{lll}
\hline & Steroids & II $S$ \\
\hline No. of patients & 5 & $7(2)^{*}$ \\
Male : female & $1: 4$ & $2: 5$ \\
Age at ouset (yr) & 42 & 44 \\
$\quad$ (range) & $(16-62)$ & $(28-61)$ \\
Duration of disease (months) & 18 & $12 \cdot 5$ \\
$\quad$ (range) & $(3-36)$ & $(3-36)$ \\
Favourable response & 4 & 6 \\
\hline
\end{tabular}

*Two patients had failed to improve on high dose steroids IIS $=$ intensive immunosuppression.

Muscle power was assessed by the MRC method. ${ }^{8}$ Electromyographic (EMG) criteria included (a) small amplitude, short duration polyphasic motor unit potentials, (b) fibrillations, positive sharp waves, increased insertional irritability, and (c) spontaneous bizarre high frequency discharge. ${ }^{9,10}$

Serum CPK levels were determined after overnight bedrest.

\section{BIOPSY DETAILS}

Biopsies were obtained with a Bergstrom needle from the vastus lateralis muscle and processed as described previously. ${ }^{11}$ In order to avoid possible artefacts due to EMG procedures and to ensure morphometric comparability biopsies were always taken on the right and EMG on the left thigh. Routine stains included haematoxylin-eosin

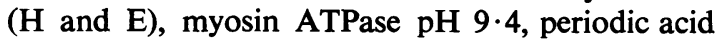
Schiff (PAS), PAS after diastase, reduced nicotinamide-adenosine dinucleotide tetrazolium reductase (NADH-TR), and oil red $O$. The biopsies were assessed conjointly by two of us (G.S. and H.A.S.) using a semiquantititave 4-point scale $(0$ to +++$)$ to grade morphological changes.

Any normal, pyknotic, or vesicular nucleus not lining the sacrolemmal membrane in a normal, degenerating, or regenerating fibre was regarded as an internal nucleus. The fibres with internal nuclei were counted independently by two observers (P.W. and H.A.S.) in 200 transversely cut fibres. In addition, a further count was made in at least $50 \%$ of the transversely cut fibres in the whole biopsy. The mean of the 3 values was taken and expressed as the percentage of fibres with internal nuclei, irrespective of whether 1 or several internal nuclei were present in a muscle fibre.

The mean diameter of at least 100 fibres of each type was measured on the ATPase stain by one of us (H.A.S.) with a computer-assisted microscope (MAGISCAN image Analysing System, Joyce-Loebl Ltd.). Atrophy and hypertrophy factors as well as variability coefficients for each fibre type were calculated as proposed by Dubowitz and Brooke 
(p. 77). ${ }^{12}$ On the same preparation the proportion of type I and type II fibres was determined.

The histological and histomorphometric features were measured without knowledge of the patient's clinical state and then correlated with sex, age at onset, duration of disease, muscle power, EMG changes, serum CPK levels, diagnostic catagory, treatment prior to the biopsy, and response to subsequent treatment.

\section{Results}

All the biopsies provided sufficient material for full histological and morphometric assessment. Minute ice crystal artefacts in 2 biopsies did not interfere with morphometry.

Inflammatory and structural changes

Inflammatory cells

Necrosis

Phagocytosis

Fibre - splitting

Basophilia

Internal nuclei

Target fibres

Motheaten fibres

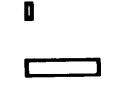

Ring fibres

Vacuolation

Lipid excess in fibres

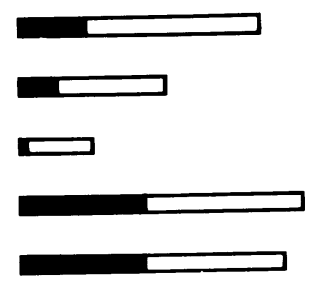

Fibrosis

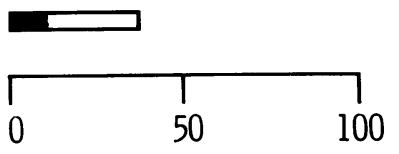

$\%$ of biopsies $(n=30)$

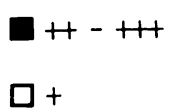

Fig. 1 Inflammatory and structural changes in the initial biopsies.
INITIAL BIOPSIES

The histological findings in the 30 initial biopsies are summarised in Fig. 1. Inflammatory infiltrates were diffuse or focal, sometimes confined to the peri- and endomysium, but usually interspersed between the individual muscle fibres as well. The infiltrates consisted largely of small mononuclear cells, occasional plasma cells, histiocytes, and polymorphonuclear leucocytes. The inflammatory changes were less marked in longstanding disease. but the difference was not significant. One patient still showed a mild, diffuse inflammatory infiltrate despite $80 \mathrm{mg}$ prednisolone per day for 18 months, but like all the other patients on steroids did not differ from the untreated group as regards necrosis, degenerative, and regenerative changes.

An excess of interstitial fibrous tissue was noted in each of 3 cases with thrombotic occlusion of capillaries and venules. However, some biopsies with interstitial fibrosis showed no vascular involvement. Among the 4 children 3 showed mild to marked interstitial inflammation, 1 of them with perivascular infiltrates and marked fibrosis. No arteritis and no arterial intimal hyperplasia was noted in the childrens' biopsies.

The changes in fibre size and distribution are shown in Fig. 2. Selective atrophy of type I fibres was seen in 4 cases; one of them had a malignancy and none were on steroids. Selective type II atrophy occurred in 8 patients, 6 of them on steroids and 3 with a malignancy. No group atrophy or fibre type grouping was seen. One patient (no. 7) with abnormal hypertrophy factors for both fibre types fulfilled all the criteria for PM. Hypertrophy factors for type ] and II in the initial biopsy were 570 and 400 , respectively. In the second biopsy (at the start of intensive immunosuppression) they were 540/540 and decreased subsequently to normal values. The fibre size ranged from $20-110 \mu \mathrm{m}$ in type $\mathrm{I}$ and $10-100 \mu \mathrm{m}$ in type II fibres.

No signs of inflammation, necrosis, degeneration or regeneration were found in 3 biopsies $(10 \%)$, but 2 of these showed marked atrophy of both fibre types; thus, only 1 biopsy in the whole series of initial biopsies showed no morphological abnormalities. This was obtained from a 24-year-old male with proximal symmetrical muscle pain and weakness for 2 months, marked 'myositic' EMG changes, and a raised serum CPK level.

The analysis of the biopsy findings in the various subgroups (see Table 1) revealed no significant intergroup differences. Apart from a greater frequency of selective type II atrophy in patients on corticosteroids there was no difference between primary and secondary referrals. No correlation between any morphological feature and the age at 
Changes in fibre size and distribution

Small angular fibres

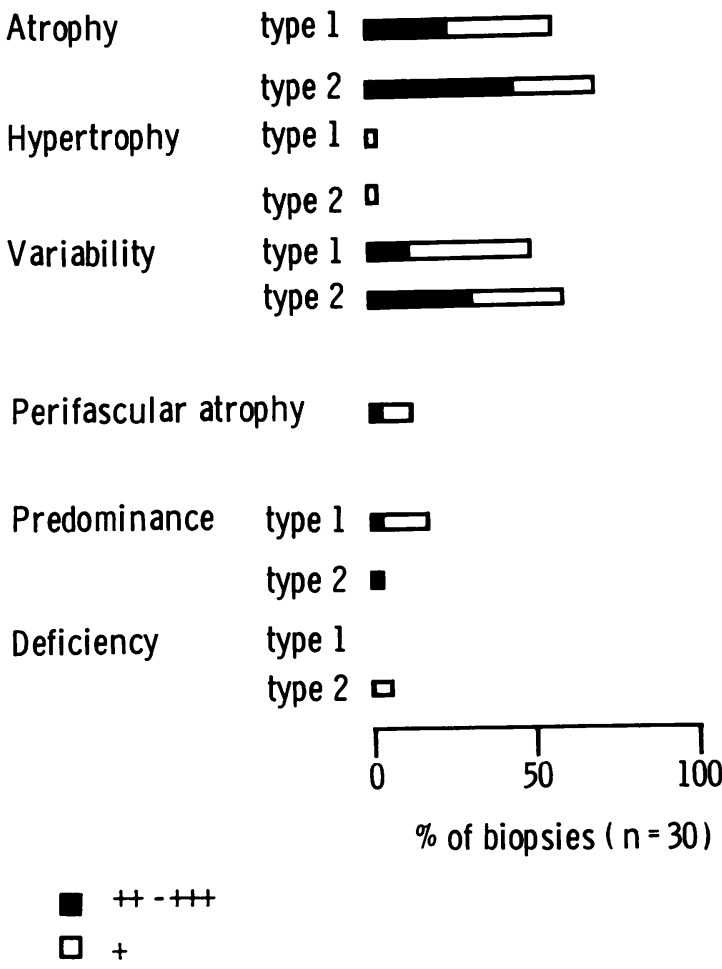

Fig. 2 Changes in fibre size and distribution in the initial biopsies.

onset, sex, duration of disease, muscle power, muscle tenderness, EMG changes, or serum CPK levels could be established.

Grouping the patients retrospectively as 'responders' and 'nonresponders' according to the effect of $60 \mathrm{mg}$ prednisolone per day or less (adult dose), we noted that the 'responders' had a much lower percentage of fibres with internal nuclei in their initial biopsies than the 'nonresponders' $(P<0.001$ Mann-Whitney $U$ test; Fig. 3). The percentage of fibres with internal nuclei did not correlate with any clinical features nor with previous low-dose corticosteroid treatment. Patients with malignancies, those who died shortly after the diagnosis had been made, and the patient with hypogammaglobulinaemia were excluded from this analysis.
Initial biopsies

Fibres with internal nuclei per 100 fibres
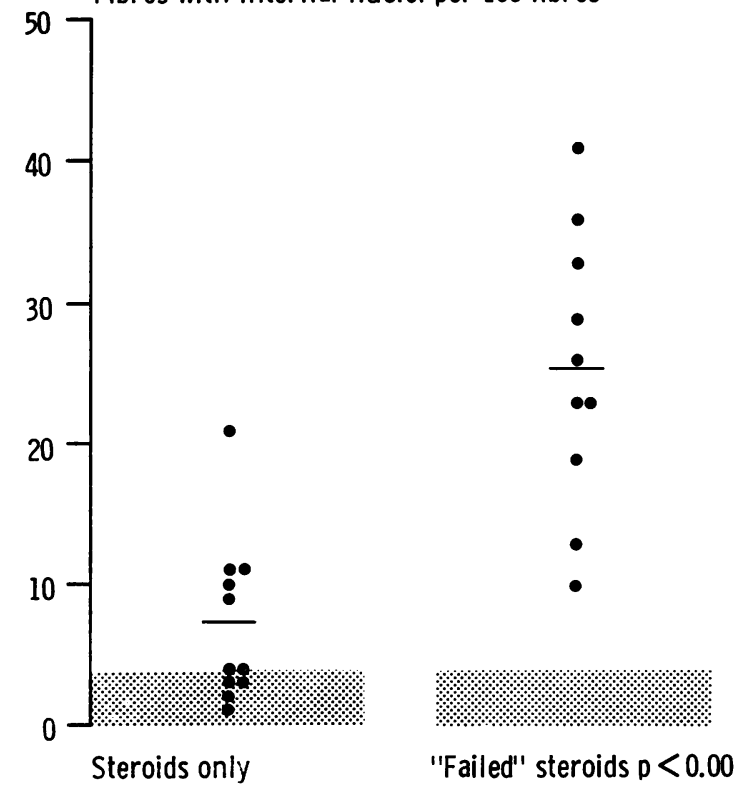

Fig. 3 Number of fibres with internal nuclei per 100 fibres in the initial biopsies; mean and normal range (dotted area).

\section{SEQUENTIAL BIOPSIES}

The histological changes in 9 patients after 4-11 months of treatment with either corticosteroids or intensive immunosuppression are shown in Fig. 4. One patient (no. 7), who showed continuing clinical deterioration after 4 months of $60 \mathrm{mg}$ prednisolone per day, rebiopsied before being started on intensive immunosuppression and again after 4 months, thus appearing in both groups. The 2 patients (nos. 3 and 5) who underwent rebiopsy after only a 1-month interval are not included in these results. The changes in atrophy factors for both fibre types, the sequential changes in internal nuclei, and the serum CPK levels, EMG changes and muscle strength at the time of the rebiopsy are shown in Table 4.

Several patients underwent further biopsies because of problems in their management. In some of these cases the different parameters varied considerably in the course of the disease. Table 4 summarises the findings in all the sequential biopsies of the 11 patients together with clinical features. In one patient (no. 2) a change from selective type II atrophy to selective type $I$ atrophy was seen under intensive immunosuppression; in 3 patients selective type II atrophy was seen after an initial atrophy involving both fibre types. 
Inflammatory and structural changes before and after corticosteroids

Inflammatory cells

Necrosis

Phagocytosis

Fibre splitting

Basophilia

Internal nuclei

Target fibres

Motheaten fibres

Ring fibres

Vacuolation

Lipid excess in fibres

Fibrosis

before
after
$\square+$

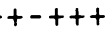

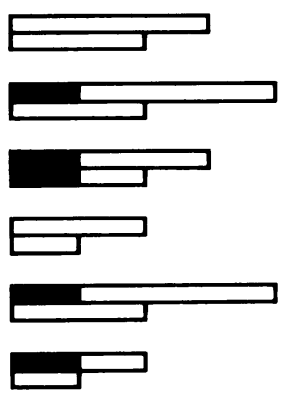

I

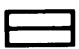

I
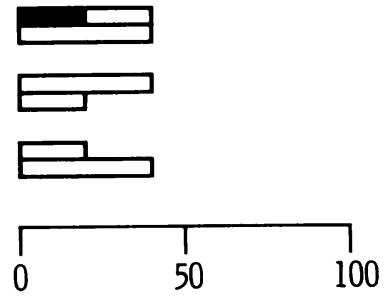

$\%$ of biopsies $(n=5)$

Fig. 4a Inflammatory and structural changes before and after at least 4 months of corticosteroids.

Although there was a marked tendency for improvement in each parameter when the patients were considered as a group, there was no significant correlation between type I and type II atrophy factors and variability coefficients in individual patients, nor between any of these and EMG changes, CPK levels, or muscle strength. In those patients with an initial increase of internal nuclei subsequent improvement in muscle power was accompanied by a decrease in internal nuclei (Table 4).

\section{Discussion}

In this series the age, sex ratio, and proportions of the diagnostic subgroups are in close agreement with Bohan et $\mathrm{al}^{3}$ and in good overall agreement with 2 other large series, ${ }^{2,13}$ in which, however, less stringent

Inflammatory and structural changes before and after immunosuppression

Inflammatory cells

Necrosis

Phagocytosis

Fibre splitting

Basophilia

Internal nuclei

Target fibres

Motheaten fibres
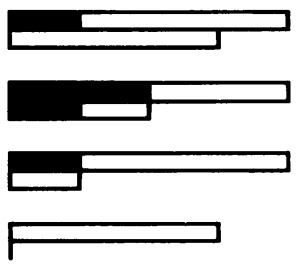

Ring fibres

Vacuolation

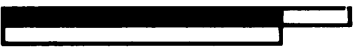

Lipid excess in fibres

Fibrosis

\section{before
after}
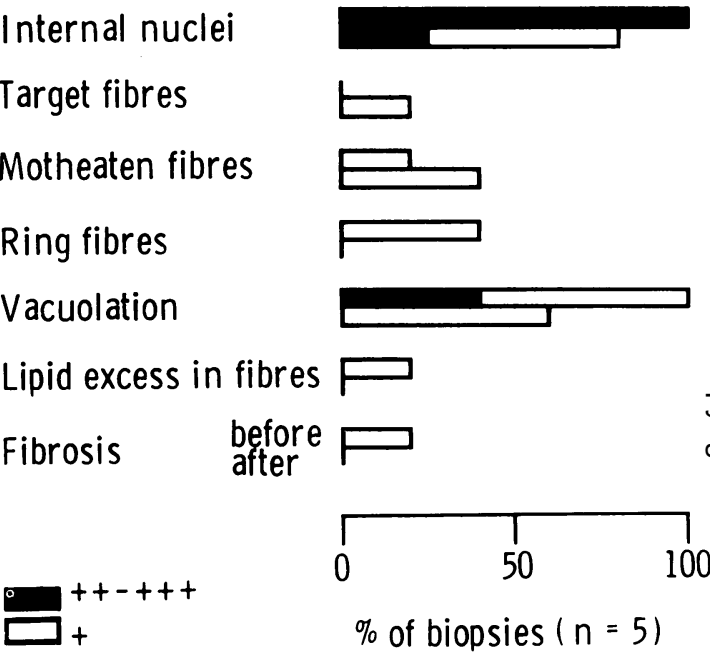

$\%$ of biopsies $(n=5)$

Fig. 4b Inflammatory and structural changes before and after at least 4 months of intensive immunosuppression.

diagnostic criteria and different classifications were used.

Needle biopsy of the vastus lateralis muscle proved to be a sensitive diagnostic indicator in the present series (Table 2). The incidence of normal biopsies in this series is less than in other reports ${ }^{2,3,14}$ if histological changes only are taken as parameters. Addition of morphometric parameters still further enhances the sensitivity of the muscle biopsy as a diagnostic tool in PM and DM, yielding a negative result in only 1 patient $(3.3 \%)$ in our series.

In quantitation of biopsy findings the question of sampling errors, variability, and inter- and intraobserver variations emerges. While no formal study has been undertaken to assess the inter- and intraobserver error for the semiquantitative histological parameters, 18 fibre size measurements carried out by two of us (P.W. and H.A.S.), each measuring 3 
Table 4 Histological, morphometric, and clinical findings in 11 patients in therapeutic study

\begin{tabular}{|c|c|c|c|c|c|c|c|c|c|c|c|c|}
\hline Patient & Sex & Age & Therapy $\mathrm{a}$ & $\begin{array}{l}\text { Intervalb } \\
\text { (months) }\end{array}$ & $\begin{array}{l}\text { Atrophy factor } \\
\text { (type I/II) }\end{array}$ & $\begin{array}{l}\text { Int. } \\
\text { nuclei }^{\mathrm{d}}\end{array}$ & Inflamm. & $\begin{array}{l}\text { Necrosis } \\
\text { degen. }\end{array}$ & Regen. & $\begin{array}{l}C P K^{\mathrm{e}} \\
(I U / L)\end{array}$ & $E M G \mathrm{f}$ & $\begin{array}{l}\text { Muscle } \\
\text { power' }\end{array}$ \\
\hline \multirow[t]{2}{*}{1} & $\mathbf{F}$ & 44 & $\mathbf{S}$ & $1 \cdot 5$ & $\begin{array}{r}950 / 810 \\
1420 / 900\end{array}$ & $\begin{array}{l}29 \\
16\end{array}$ & $\begin{array}{l}+ \\
+\end{array}$ & $\stackrel{+}{++}$ & $\begin{array}{l}++ \\
++\end{array}$ & $\begin{array}{l}1516 \\
1656\end{array}$ & +++ & $\begin{array}{ll}3 & 14 \\
4 & 14\end{array}$ \\
\hline & & & IIS & 6 & $280 / 390$ & 12 & + & 0 & & 416 & + & $4+14+$ \\
\hline \multirow[t]{3}{*}{2} & $\mathbf{F}$ & 57 & & & $180 / 490$ & 23 & 0 & $\mathbf{0}$ & ++ & 59 & + & $4 \quad / 3$ \\
\hline & & & IIS & 3 & $290 / 110$ & 13 & 0 & 0 & 0 & 132 & & $4 \quad / 4$ \\
\hline & & & IIS & 7 & $450 / 130$ & 14 & 0 & 0 & + & 258 & & $4+14+$ \\
\hline \multirow[t]{2}{*}{3} & $\mathbf{M}$ & 28 & $\mathbf{S}$ & & $940 / 1430$ & 36 & + & + & + & 42 & + & $4 \quad / 4$ \\
\hline & & & IIS & 1 & $1870 / 1880$ & 49 & +++ & +++ & ++ & 60 & + & $\begin{array}{l}4 \quad / 4+ \\
(5 / 5)\end{array}$ \\
\hline \multirow[t]{4}{*}{4} & $\mathbf{F}$ & 61 & & & $450 / 420$ & 23 & ++ & ++ & ++ & 271 & + & $4 \quad / 4$ \\
\hline & & & & 4 & $10 / 300$ & 15 & ++ & ++ & + & 192 & & $4 \quad 14$ \\
\hline & & & IIS & 8 & $620 / 720$ & 14 & + & + & +++ & 232 & & $4+/ 4$ \\
\hline & & & IIS & 20 & $50 / 500$ & 24 & + & ++ & + & 104 & + & $3+/ 3+$ \\
\hline \multirow[t]{2}{*}{5} & $\mathbf{F}$ & 56 & & & $120 / 700$ & 13 & + & + & + & 180 & +++ & $4 \quad / 4$ \\
\hline & & & IIS & 1 & $40 / 710$ & 1 & + & 0 & + & 14 & ++ & $4+14+$ \\
\hline \multirow[t]{4}{*}{6} & F & 32 & & & $1530 / 1660$ & 33 & + & ++ & ++ & 168 & +++ & $4 \quad 14$ \\
\hline & & & IIS & 2 & $210 / 1440$ & 5 & 0 & 0 & ++ & 160 & ++ & $4+14+$ \\
\hline & & & IIS & 6 & $920 / 1040$ & 10 & + & ++ & ++ & 83 & no & $4+15$ \\
\hline & & & IIS & 12 & $30 / 210$ & 3 & 0 & 0 & 0 & 209 & & \\
\hline \multirow[t]{4}{*}{7} & $\mathbf{M}$ & 29 & & & $30 / 190$ & 41 & + & + & + & 1760 & +++ & $4 \quad / 4$ \\
\hline & & & $\mathbf{S}$ & 5 & $0 / 10$ & 53 & + & + & + & 240 & +++ & $4 \quad / 4$ \\
\hline & & & IIS & 1 & $90 / 80$ & 50 & + & + & ++ & 200 & +++ & $4 \quad / 4$ \\
\hline & & & IIS & 3 & $20 / 190$ & 13 & + & + & + & 65 & no & $5 \quad 15$ \\
\hline \multirow[t]{2}{*}{8} & $\mathbf{F}$ & 62 & $\mathbf{S}$ & & $80 / 510$ & 3 & + & + & ++ & 1480 & no & $414+$ \\
\hline & & & $\mathbf{S}$ & 15 & $80 / 480$ & 4 & + & + & + & 920 & & $4+14+$ \\
\hline \multirow[t]{2}{*}{9} & $\mathbf{F}$ & 16 & & & $10 / 40$ & 2 & 0 & + & + & 1280 & +++ & $3 / 3$ \\
\hline & & & $\mathbf{S}$ & 12 & $190 / 180$ & 3 & 0 & 0 & 0 & 252 & & $\begin{array}{l}4 / 4+ \\
(5 / 5)\end{array}$ \\
\hline \multirow[t]{2}{*}{10} & $\mathbf{F}$ & 40 & & & $290 / 1510$ & 1 & 0 & 0 & 0 & 7 & ++ & $4+14+$ \\
\hline & & & $\mathbf{S}$ & 16 & $100 / 420$ & 1 & 0 & 0 & 0 & 16 & & $5 / 5$ \\
\hline \multirow{3}{*}{11} & $\mathbf{F}$ & 61 & & & $310 / 1130$ & 11 & + & + & ++ & 644 & +++ & $4 \quad / 4$ \\
\hline & & & $\mathbf{S}$ & 1 & $140 / 570$ & 9 & + & + & $\mathbf{0}$ & 244 & & $4+14+$ \\
\hline & & & $\mathbf{S}$ & 6 & $110 / 460$ & 2 & 0 & 0 & 0 & 33 & & 515 \\
\hline
\end{tabular}

a Therapy: Treatment since previous biopsy or at the time of the first biopsy.

b Interval: Since previous biopsy.

c Atrophy factor: Type I, normal: male $<150$ female $<100$; type II, normal: male $<150$ female $<200$.

a Internal nuclei: Percentage of fibres with internal nuclei.

e CPK: Normal $<80 \mathrm{IU} / 1$.

f EMG: For parameters see text; no $=$ normal.

guscle power: Proximal upper limbs/proximal lower limbs: values in brackets $=$ after 1 year of treatment $5=$ normal.

samples of at least 200 fibres thrice, showed an inter- and intraobserver variation of less than $1 \%$ (Altman D, Slavin G, unpublished data).

Histological data are comparable only if the duration and the activity of the disease at the time of the biopsy or necropsy are known. Yet the reports on large series mentioned neither of them. ${ }^{2,3,13}$ In our initial biopsies inflammatory infiltrates did not correlate with the duration of disease or the use of low dose corticosteroids. While inflammatory changes in the biopsy were a prerequisite in 20 patients with PM reported by Riddoch and MorganHughes, ${ }^{15}$ Munsat and Cancilla ${ }^{14}$ described 16 patients with clinical evidence of PM and definite pathological alterations in their biopsies but no inflammation at all. The mean duration of disease in the former group was $\mathbf{3 3}$ months compared with $\mathbf{1 0}$ months in the latter.

Inflammatory infiltrates in PM and DM associated with malignancies have been said to be sparse relative to necrosis, degeneration, and regeneration. ${ }^{16,17}$ These observations, however, were not supported by the data of Bohan et al. ${ }^{3}$ In the 4 cases associated with malignant disease in the present series the most prominent feature was marked vacuolation of both fibre types on the ATPase stain in 3, with few other degenerative or regenerative changes. Two biopsies showed mild to moderate inflammation.

Perivascular infiltrates and thrombosis mainly involved venules and capillaries. When thrombosis occurred, interstitial fibrosis was more marked. From studies of muscles after experimental venous or arterial occlusion in animals it would appear that fibrosis results from venous occlusion with maintenance of the arterial blood supply. ${ }^{18}$

Banker and Victor ${ }^{19}$ in their post-mortem study of 8 children with DM drew attention to widespread angiitis, arterial intimal hyperplasia, and thrombosis in childhood DM with subsequent infarction of muscles and nerves. These observations have been confirmed by Carpenter et al. ${ }^{20}$ Biopsies of the 4 children in our series did not show any arteritis or intimal hyperplasia of arterioles nor was there 
children in our series did not show any arteritis or muscle infarction or denervation atrophy. This is in agreement with the findings in the 11 children in Bohan et al.'s series, ${ }^{3}$ indicating that severe vasculitis as described by Banker and Victor ${ }^{19}$ in their postmortem specimens is not necessarily seen in the small biopsy sample.

Regenerative changes, namely, basophillia of small muscle fibres and large, vesicular nuclei with prominent nucleoli, were the most frequent finding in both the initial and the sequential biopsies. This is at variance with Brooke and Kaplan ${ }^{4}$ and Bohan et al., ${ }^{3}$ who noted an incidence of regeneration of $30 \%$ and $48 \%$ respectively, and Riddoch and Morgan-Highes' statement ${ }^{15}$ that regenerating fibres were rarely seen in patients with a history of more than 1 year. In our series regeneration tended to become less marked with treatment, yet actually disappeared in 2 of the 11 patients only. While regeneration was present in all 8 patients of Mastaglia and Kakulas, ${ }^{6}$ the subsarcolemmal crescentic myoblasts described in their series were not seen in our biopsies. This may be due to our exclusive use of frozen sections as opposed to the thinner paraffin sections used by these authors.

Perifascicular atrophy, regarded as a highly specific feature of PM and $D M,{ }^{14,19}$ is also found in inflammatory myopathies with mixed connective tissue disease ${ }^{21}$ and said to occur in myositis associated with systemic lupus erythematosus. ${ }^{22}$ In both instances the muscle disorder might not necessarily fulfil the criteria for PM or DM as proposed by Bohan and Peter. ${ }^{7}$ Perifascicular atrophy appeared less frequently in the present $(13 \%)$ than in other series. ${ }^{4,14}$ The patient with SLE did not show it; perifascicular atrophy was most striking in a 7-year-old Indian girl with a 4-year history of severe DM treated with high doses of prednisolone for most of the time.

Fibre type I predominance as defined by Dubowitz and Brooke (p. 17) ${ }^{12}$ was seen in 5 cases, which is in agreement with other reports. ${ }^{4,14}$ The 1 patient with type II predominance had been treated with $80 \mathrm{mg}$ prednisolone per day for 18 months. The significance of these findings is difficult to assess. ${ }^{23}$

Changes in muscle fibre size are associated with many disorders. Selective type I atrophy has been described in dystrophia myotonica, rheumatoid arthritis, myotubular myopathy, nemaline myopathy, congenital fibre type dysproportion, and PM/DM. ${ }^{12}$ The incidence of $13 \%$ selective type I atrophy in this series is in keeping with Brooke and Kaplan, ${ }^{4}$ though Munsat and Cancilla ${ }^{14}$ did not see it in their 16 patients. Selective type II atrophy is commonly attributed to disuse of a muscle, but occurs in a wide range of metabolic disorders including corticosteroid treatment. ${ }^{12}$ In 10 patients responding to either corticosteroids alone or intensive immunosuppression the atrophy of type II fibres decreased despite the rather high dose of steroids in both regimens over a prolonged period of time (Table 4). The only increase in type II atrophy occurred in the patient (no. 4) who did not respond to intensive immunosuppression. This suggests that corticosteroids, although decreasing muscle protein synthesis in themselves, ${ }^{24}$ tip the balance towards anabolism in 'responders' by suppressing the catabolic disease activity, while they enhance catabolism in 'nonresponders'. In the differential diagnosis between continuing disease activity and corticosteroid myopathy, both of which decrease muscle power, selective type II atrophy in the absence of inflammation would favour the latter diagnosis. Discrete degenerative and regenerative changes would not necessarily be helpful, since they can occur in both conditions. ${ }^{18}$

In contrast to muscular dystrophies, which may otherwise mimic the histological features of PM and DM, hypertrophy of muscle fibres is usually lacking in the latter conditions. ${ }^{12}$ Nevertheless, in the 1 patient (no. 7) with abnormal hypertrophy factors of both fibre types we found no evidence for a dystrophic process. He responded well to intensive immunosuppression, hypertrophy factors and muscle power returning to normal.

While Carpenter et al. ${ }^{20}$ correlated certain histological features rather summarily with diagnostic subgroups in 20 patients with idiopathic inflammatory myopathies, there were no significant histological differences in the present and in another series. $^{3}$ Since muscle tissue has only a restricted number of reactions to injury, different pathogenetic mechanisms can result in the same morphological features. Moreover, in PM and DM subgroup differences might not emerge because of the small numbers in each group, due to sampling errors or because of other heterogeneities within the subgroups.

No consistent correlation between histological features and clinical parameters has been found in previous series.,13 Morphometric studies of fibre diameters and associated atrophy or hypertrophy factors in this series are similarly unhelpful. This lack of correlation may be due to the wide variability of each parameter or to their relative lack of sensitivity and/or specificity. The difficulties encountered can be illustrated by the problems in measuring muscle strength, which is the most reliable clinical yardstick. Apart from the rather crude methods used for assessing muscle power, ${ }^{8}$ which are particularly inaccurate when applied to proximal muscle groups, the muscle power depends on subjective factors like 
motivation and pain, and physiological modalities such as muscle bulk, energy supply, electrolyte concentrations, and the contractile proteins.

However, in those patients with an initially increased number of internal nuclei sequential biopsy shows that an improving muscle power is matched by reversion of the internal nuclear count. Internal nuclei are increased in many myopathies, notably in myotubular myopathy, myotonic dystrophy, hypothyroidism, and corticosteroid myopathy, but also in chronic peripheral neuropathies such as Charcot-Marie-Tooth disease. ${ }^{12}$ The decrease of initially elevated numbers of internal nuclei with response to treatment has recently been reported in myopathy due to hypothyroidism. ${ }^{11}$ In contrast to our findings the central nuclei were largely confined to type II fibres which also showed selective atrophy.

There is a striking difference in the percentage of fibres with internal nuclei in the initial biopsy between 'responders' and 'nonresponders' to $60 \mathrm{mg}$ prednisolone per day or less (adult dose). Internal nuclei may be an indicator for both degenerative and regenerative processes and thus parallel the overall severity of structural muscle fibre damage in these diseases.

Hence clinical response to corticosteroids in PM and DM seems to be inversely correlated with the extent of muscle fibre damage as indicated by the percentage of fibres with internal nuclei in the initial biopsy. Because an indicator for corticosteroid responsiveness would be of great clinical value, this observation needs further confirmation in a larger series of patients. Preliminary results in a further 12 patients seem to confirm the strong inverse correlation between responsiveness to corticosteroids and the percentage of fibres with internal nuclei.

We thank Dr D. S. Smith for the EMG studies, Mr D. Altman for statistical advice, Dr A. M. Denman for the case histories of his patients, and the physiotherapists for assessing the muscle power of the patients.

H. A. Schwarz was supported by a grant from the Swiss Academy of Medical Sciences.

\section{References}

1 Edwards R H T. Percutaneous needle-biopsy of skeletal muscle in diagnosis and research. Lancet 1971; 2 : 593-6.

2 DeVere R, Bradley W G. Polymyositis: Its presentation, morbidity and mortality. Brain 1975;98: 637-66.
3 Bohan A, Peter J B, Bowman R L, Pearson C M. A computer-assisted analysis of 153 patients with polymyositis and dermatomyositis. Medicine 1977; 56: 255-86.

4 Brooke M H, Kaplan H. Muscle pathology in rheumatoid arthritis, polymyalgia rheumatica and polymyositis. Arch Pathol 1972; 94: 101-18.

5 Denman A M, Kinsley M, Partridge C, et al. The treatment of connective tissue diseases with antilymphocyte globulin. Postgrad Med J 1976; 52 (suppl. 5): 118-21.

- Mastaglia F L, Kakulas B A. A histological and histochemical study of skeletal muscle regeneration in polymyositis. J Neurol Sci 1970; 10: 471-87.

7 Bohan A, Peter J B. Polymyositis and deramtomyositis. N Eng J Med 1975; 292: 344-7; 403-7.

8 British Medical Research Council. Aid to the Investigation of Peripheral Nerve Injuries, 2nd ed. London HMSO, 1943.

- Lambert E H, Sayre G B, Eaton L M. Electrical activity of muscle in polymyositis. Trans Am Neurol Ass 1954; 79: 64-9.

10 Marinacci A A. Electromyography in the diagnosis of polymyositis. Electromyography $1965 ; 5: 255-68$.

11 McKeran R O, Ward P. Slavin G, Paul E A. Central nuclear counts in muscle fibres before and during treatment in hypothyroid myopathy. J Clin Path 1979; 32: 229-33.

12 Dubowitz V, Brooke M H. Muscle Biopsy. London: Saunders, 1973.

13 Winkelmann R K, Mulder D W, Lambert E H. Howard F M, Diesser G R. Comparison of untreated and cortisonetreated patients. Mayo Clin Proc 1968; 43: 545-56

14 Munsat T, Cancilla P. Polymyositis without inflammation. Bull Los Angeles Neurol Soc 1974; 39: 113-20.

15 Riddoch D, Morgan-Hughes J A. Prognosis in adult polymyositis. J Neurol Sci $1975 ; 26$ : 71-80.

16 Smith B. Skeletal muscle necrosis associated with carcinoma. J Pathol 1969; 97: 207-10.

17 Urich $H$. Wilkinson $M$. Necrosis of muscle with carcinoma: myositis or myopathy? $J$ Neurol, Neurosurg Psychiatry 1970; 33: 398-407.

18 Adams R D. Diseases of Muscle, 3rd ed. Hagerstown: Harper and Row, 1975.

19 Banker B Q, Victor M. Dermatomyositis (systemic angiopathy) of childhood. Medicine 1966; 45: 261-89.

20 Carpenter S, Karpati G, Eisen A. A morphologic study in polymyositis: clues to pathogenesis of different types. In: Bradley W G, Gardner-Medwin D, Walton J N. eds. Recent Advances in Myology, Amsterdam: Excerpta Medica, 1975; 374-7.

21 Oxenhandler R, Hart M, Corman L, Sharp G, Adelstein E. Pathology of skeletal muscle in mixed connective tissue disease. Arthritis Rheum 1979; 20: 985-8.

22 Adams R D. The pathologic substratum of polymyositis. In: Pearson C M, Mostoti F K. eds. The Striated Muscle. Baltimore: Williams and Wilkins, 1973; 299.

23 Jennekens F G I, Tomlinson B E, Walton J N. Data on the distribution of fibre types in 5 human limb muscles. $J$ Neurol Sci 1971; 14: 245-257.

24 Pennington R J T. Aspects of the biochemistry of the muscular dystrophies. In: Lundt G G, Marchbanks R M, eds. Biochemistry of Myasthenia Gravis and Muscular Dystrophy. London: Academic Press, 1978; 234. 